\title{
DIAGONAL RICCATI STABILITY OF A CLASS OF TIME-DELAY SYSTEMS
}

\author{
Alexander Aleksandrov \\ Saint Petersburg State University \\ Russia \\ a.u.aleksandrov@spbu.ru
}

Article history:

Received 13.10.2018, Accepted 19.12.2018

\begin{abstract}
A complex system describing interaction of subsystems of the second order with delay in connections between them is studied. Necessary and sufficient conditions of the existence of a diagonal LyapunovKrasovskii functional for the considered system are derived. The obtained results are applied for the stability analysis of a mechanical system and a model of population dynamics. In addition, it is shown that they can be used in a problem of formation control.
\end{abstract}

\section{Key words}

Time-delay system, Lyapunov-Krasovskii functional, diagonal stability, population dynamics, multiagent system.

\section{Introduction}

Diagonal Lyapunov functions is a powerful tool for the stability analysis of wide classes of systems [Kaszkurewicz and Bhaya, 1999]. On the one hand, this is due to the fact that they possess a simple structure. On the other hand, for many types of nonlinear systems, the problem of constructing Lyapunov functions naturally results in choosing a Lyapunov candidate function in a diagonal form. It should be noted that diagonal Lyapunov functions are especially often used for the stability investigation of complex systems, neural networks and models of population dynamics [Hofbauer and Sigmund, 1998; Kaszkurewicz and Bhaya, 1999; Arcat and Sontag, 2006; Aleksandrov, Aleksandrova and Platonov, 2013; Talagaev, 2017; Alyshev, Dudarenko and Melnikov, 2018]. Moreover, in many cases, with the aid of such functions it is possible to derive not only sufficient, but also necessary stability conditions [Kaszkurewicz and Bhaya, 1999; Shorten

\author{
Nadezhda Kovaleva \\ Saint Petersburg State University \\ Russia \\ nadia.kv13@gmail.com
}

and Narendra, 2009].

The problem of the existence of diagonal Lyapunov functions is well investigated for linear time-invariant systems of differential and difference equations. In [Kraaijevanger, 1991; Kaszkurewicz and Bhaya, 1999; Arcat and Sontag, 2006; Mason and Shorten, 2006; Shorten and Narendra, 2009], conditions are obtained under which quadratic Lyapunov functions with diagonal matrices can be constructed for these systems. A linear system possessing such a Lyapunov function is called diagonally Lyapunov stable [Mason and Shorten, 2006].

In [Mason, 2012], the problem of diagonal Riccati stability was stated. A linear positive differential system with a constant delay was considered, and conditions guaranteeing that the system admits a diagonal Lyapunov-Krasovskii functional were investigated. The results of [Mason, 2012] have got further development in [Aleksandrov and Mason, 2014; Aleksandrov and Mason, 2016; Aleksandrov and Mason, 2018]. In particular, in [Aleksandrov and Mason, 2016], a criterion of diagonal Riccati stability was obtained for linear time-invariant difference-differential systems of a general form (not necessary for positive ones). However, it is worth mentioning that the criterion is insufficiently constructive. Therefore, an interesting and important problem is that of finding classes of time-delay systems for which constructively verifiable conditions of diagonal Riccati stability can be derived. Some such classes both linear and nonlinear systems were determined in [Aleksandrov and Mason, 2016; Aleksandrov, Mason and Vorob'eva, 2017; Aleksandrov and Mason, 2018].

In the present contribution, a nonlinear time-delay system is studied. The system describes the interaction of subsystems of the second order and possesses a special structure of connections between the subsystems. We will look for conditions of the existence of a diagonal Lyapunov-Krasovskii functional of a prescribing form for the considered system. Moreover, we 
will show that such conditions can be used for the stability analysis of a mechanical system and a model of population dynamics and for the design of a protocol providing equidistant deployment of mobile agents on a line segment.

\section{Statement of the Problem}

Consider the time-delay system

$$
\dot{x}(t)=A f(x(t))+B f(x(t-\tau)) .
$$

Here $x(t)=\left(x_{1}(t), \ldots, x_{n}(t)\right)^{T}$ is the state vector, $A=\left\{a_{i j}\right\}_{i, j=1}^{n}$ and $B=\left\{b_{i j}\right\}_{i, j=1}^{n}$ are constant matrices, $f(x)=\left(f_{1}\left(x_{1}\right), \ldots, f_{n}\left(x_{n}\right)\right)^{T}$, where scalar functions $f_{j}\left(x_{j}\right)$ are continuous for $\left|x_{j}\right|<H(0<$ $H \leq+\infty)$ and belong to a sector-like constrained set defined as follows: $x_{j} f_{j}\left(x_{j}\right)>0$ for $x_{j} \neq 0$, $j=1, \ldots, n, \tau$ is a constant nonnegative delay.

The system (1) is well-known Persidskii type system, see [Kaszkurewicz and Bhaya, 1999]. Such systems are widely used for the modeling automatic control systems and neural networks.

Let $\mathbb{R}^{n}$ denote the $n$-dimensional Euclidean space, $\|\cdot\|$ be the Euclidean norm of a vector, $C\left([-\tau, 0], \mathbb{R}^{n}\right)$ be the space of continuous functions $\varphi(\theta):[-\tau, 0] \rightarrow \mathbb{R}^{n}$ with the uniform norm $\|\varphi\|_{\tau}=\sup _{\theta \in[-\tau, 0]}\|\varphi(\theta)\|$, and $\Omega_{H}$ be the set of functions $\varphi(\theta) \in C\left([-\tau, 0], R^{n}\right)$ such that $\|\varphi\|_{\tau}<H$. In addition, let $x\left(t, t_{0}, \varphi\right)$ stand for a solution of (1) with the initial conditions $t_{0} \geq 0$, $\varphi(\theta) \in \Omega_{H}$, and $x_{t}\left(t_{0}, \varphi\right)$ denote the restriction of the solution to the segment $[t-\tau, t]$, i.e., $x_{t}\left(t_{0}, \varphi\right): \theta \rightarrow$ $x\left(t+\theta, t_{0}, \varphi\right), \theta \in[-\tau, 0]$. When the initial conditions are not important, or are well defined from the context, we will write $x(t)$ and $x_{t}$, instead of $x\left(t, t_{0}, \varphi\right)$ and $x_{t}\left(t_{0}, \varphi\right)$, respectively.

Definition 1. The system (1) is called diagonally Riccati stable if there exist diagonal positive definite matrices $P$ and $Q$ such that the matrix

$$
R=A^{T} P+P A+Q+P B Q^{-1} B^{T} P
$$

is negative definite.

Remark 1. It is known, see [Aleksandrov and Mason, 2016], that if the system (1) is diagonally Riccati stable, then it admits a diagonal Lyapunov-Krasovskii functional of the form

$V\left(x_{t}\right)=\sum_{i=1}^{n}\left(2 p_{i} \int_{0}^{x_{i}(t)} f_{i}(u) d u+q_{i} \int_{t-\tau}^{t} f_{i}^{2}\left(x_{i}(\theta)\right) d \theta\right)$

where $p_{i}$ and $q_{i}$ are diagonal entries of the matrices $P$ and $Q$, respectively. It is worth mentioning that the existence of such a functional implies that the zero solution of the system (1) is asymptotically stable for an arbitrary constant nonnegative delay $\tau$, see [Mason, 2012; Aleksandrov and Mason, 2016].

In the present paper, we consider the case where $n$ is an even number ( $n=2 k$ and $k$ is a positive integer), and the matrices $A$ and $B$ possess the following structures

$$
A=\left(\begin{array}{ccccccc}
a_{11} & a_{12} & 0 & 0 & \cdots & 0 & 0 \\
a_{21} & a_{22} & 0 & 0 & \cdots & 0 & 0 \\
0 & 0 & a_{33} & a_{34} & \cdots & 0 & 0 \\
0 & 0 & a_{43} & a_{44} & \cdots & 0 & 0 \\
\vdots & \vdots & \vdots & \vdots & \ddots & \vdots & \vdots \\
0 & 0 & 0 & 0 & \cdots & a_{n-1 n-1} & a_{n-1 n} \\
0 & 0 & 0 & 0 & \cdots & a_{n n-1} & a_{n n}
\end{array}\right)
$$

$$
B=\left(\begin{array}{cccccccc}
0 & 0 & 0 & 0 & \cdots & 0 & 0 & b_{1} \\
0 & 0 & 0 & 0 & \cdots & 0 & 0 & 0 \\
0 & b_{2} & 0 & 0 & \cdots & 0 & 0 & 0 \\
0 & 0 & 0 & 0 & \cdots & 0 & 0 & 0 \\
0 & 0 & 0 & b_{3} & \cdots & 0 & 0 & 0 \\
\vdots & \vdots & \vdots & \vdots & \ddots & \vdots & \vdots & \vdots \\
0 & 0 & 0 & 0 & \cdots & b_{k} & 0 & 0 \\
0 & 0 & 0 & 0 & \cdots & 0 & 0 & 0
\end{array}\right) .
$$

Thus, (1) can be treated as a closed-loop complex system describing interaction of subsystems of the second order with delay in the connections between the subsystems, see Fig. 1.

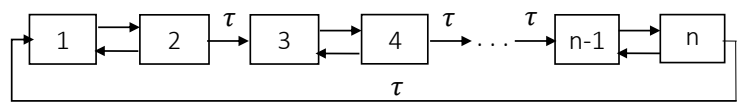

Figure 1. Structure of connections in the system (1)

We will look for conditions of diagonal Riccati stability of the system (1) with matrices (3) and (4).

Moreover, we will consider some applications of such conditions to problems of analysis and synthesis of time-delay systems.

\section{A Criterion of Diagonal Riccati Stability}

Let $P$ and $Q$ be positive definite diagonal martices with diagonal entries $p_{1}, \ldots, p_{n}$ and $q_{1}, \ldots, q_{n}$, respectively. If matrices $A$ and $B$ are defined by the formulae (3) and (4), then the matrix (2) can be rewritten 
as follows

$$
R=\left(\begin{array}{cccc}
R^{(1)} & 0 & \cdots & 0 \\
0 & R^{(2)} & \cdots & 0 \\
\vdots & \vdots & \ddots & \vdots \\
0 & 0 & \cdots & R^{(k)}
\end{array}\right)+\widetilde{R}
$$

Here $\widetilde{R}=\operatorname{diag}\left\{q_{1}, 0, q_{3}, 0, \ldots, q_{n-1}, 0\right\}$,

$$
\begin{aligned}
& R^{(s)}=\left(\begin{array}{cc}
r_{11}^{(s)} & r_{12}^{(s)} \\
r_{12}^{(s)} & r_{22}^{(s)}
\end{array}\right), \\
& r_{11}^{(s)}=2 a_{2 s-12 s-1} p_{2 s-1}+\frac{p_{2 s-1}^{2} b_{s}^{2}}{q_{2 s-2}}, \\
& r_{12}^{(s)}=a_{2 s-12 s} p_{2 s-1}+a_{2 s 2 s-1} p_{2 s}, \\
& r_{22}^{(s)}=2 a_{2 s 2 s} p_{2 s}+q_{2 s}, \quad s=1, \ldots, k,
\end{aligned}
$$

and $q_{0}=q_{n}$.

Hence, the system (1) is diagonally Riccati stable if and only if there exist positive numbers $p_{1}, \ldots, p_{n}$ and $q_{2}, q_{4}, \ldots, q_{n}$ such that the inequalities

$$
\begin{aligned}
& \left(2 a_{2 s-12 s-1} p_{2 s-1}+\frac{p_{2 s-1}^{2} b_{s}^{2}}{q_{2 s-2}}\right) \\
& \times\left(2 a_{2 s 2 s} p_{2 s}+q_{2 s}\right) \\
& >\left(a_{2 s-12 s} p_{2 s-1}+a_{2 s 2 s-1} p_{2 s}\right)^{2}, \\
& 2 a_{2 s 2 s} p_{2 s}+q_{2 s}<0, \quad s=1, \ldots, k,
\end{aligned}
$$

hold.

With the aid of (5), we obtain the necessary conditions of diagonal Riccati stability

$$
\begin{aligned}
& a_{i i}<0, \quad i=1, \ldots, n, \\
& a_{2 s-12 s-1} a_{2 s 2 s}>a_{2 s-12 s} a_{2 s 2 s-1}, \\
& s=1, \ldots, k .
\end{aligned}
$$

Assume that conditions (6) are fulfilled.

Choose some $s \in\{1, \ldots, k\}$ and consider the following cases:

(I) If $a_{2 s 2 s-1}=0$, then, for any positive numbers $q_{2 s-2}$ and $q_{2 s}$, the corresponding inequalities from (5) will be valid for sufficiently small values of $p_{2 s-1}$ and for sufficiently large values of $p_{2 s}$.

(II) If $a_{2 s 2 s-1} \neq 0, b_{s}=0$ and $a_{2 s-12 s}=0$, then, for any positive numbers $q_{2 s-2}$ and $q_{2 s}$, the corresponding inequalities from (5) will be valid for $p_{2 s}=$ $-q_{2 s} / a_{2 s} 2 s$ and for sufficiently large values of $p_{2 s-1}$.

(III) If $a_{2 s 2 s-1} \neq 0, b_{s}=0$ and $a_{2 s-12 s} \neq 0$, then, for any positive numbers $q_{2 s-2}$ and $q_{2 s}$, the corresponding inequalities from (5) will be valid for $p_{2 s-1}=p_{2 s}\left|a_{2 s} 2 s-1 / a_{2 s-12 s}\right|$ and for sufficiently large values of $p_{2 s}$.
(IV) Let $a_{2 s 2 s-1} \neq 0$ and $b_{s} \neq 0$. Consider the $s$ th pair of inequalities from the system (5). Find $p_{2 s-1}$ and $p_{2 s}$ for which the pair defines the largest domain of values for the remaining parameters. It is easy to verify that we should take

$$
\begin{gathered}
p_{2 s}=\frac{a_{2 s 2 s}}{a_{2 s 2 s-1}^{2}}\left(2 p_{2 s-1} a_{2 s-12 s-1}+\frac{p_{2 s-1}^{2} b_{s}^{2}}{q_{2 s-2}}\right) \\
-\frac{a_{2 s-12 s}}{a_{2 s 2 s-1}} p_{2 s-1}, \\
p_{2 s-1}=-\frac{q_{2 s-2} \Delta_{2 s-12 s}}{a_{2 s 2 s} b_{s}^{2}}
\end{gathered}
$$

for $\widetilde{\Delta}_{2 s-12 s}>0$, and

$$
p_{2 s-1} \rightarrow-\frac{2 a_{2 s-12 s-1} q_{2 s-2}}{b_{s}^{2}}-0
$$

for $\widetilde{\Delta}_{2 s-12 s} \leq 0$. Here

$$
\begin{aligned}
& \Delta_{2 s-12 s}=a_{2 s-12 s-1} a_{2 s 2 s}-a_{2 s-12 s} a_{2 s 2 s-1}, \\
& \widetilde{\Delta}_{2 s-12 s}=a_{2 s-12 s-1} a_{2 s 2 s}+a_{2 s-12 s} a_{2 s 2 s-1} .
\end{aligned}
$$

For such a choice of $p_{2 s-1}$ and $p_{2 s}$, the $s$ th pair of inequalities from (5) is equivalent to the condition $a_{2 s 2 s-1}^{2} b_{s}^{2} q_{2 s}<\eta_{s} q_{2 s-2}$, where

$$
\eta_{s}=\left\{\begin{array}{c}
\Delta_{2 s-12 s}^{2} \text { for } \widetilde{\Delta}_{2 \mathrm{~s}-12 \mathrm{~s}}>0 \\
-4 a_{2 s-12 s-1} a_{2 s 2 s} a_{2 s-12 s} a_{2 s 2 s-1} \\
\text { for } \widetilde{\Delta}_{2 \mathrm{~s}-12 \mathrm{~s}} \leq 0
\end{array}\right.
$$

Thus, we arrive at the following theorem.

Theorem 1. Let the matrices $A$ and $B$ in the system (1) be of the form (3) and (4), respectively. Then the system is diagonally Riccati stable if and only if the conditions (6) and

$$
\prod_{s=1}^{k} \eta_{s}>\prod_{s=1}^{k} a_{2 s 2 s-1}^{2} b_{s}^{2}
$$

are valid.

Remark 2. If there exists a number $r \in\{1, \ldots, k\}$ such that $a_{2 r 2 r-1}=0$ or $b_{r}=0$, then for the diagonal Riccati stability of (1) it is necessary and sufficient the fulfilment of the conditions (6). 


\section{Stability Analysis of a Model of Population Dy- namics}

In this section, we will show how the results described above can be applied to a generalized Lotka-Volterra model of population dynamics.

Let the system

$$
\begin{aligned}
& \dot{x}_{i}(t)=x_{i}(t)\left(c_{i}+\sum_{j=1}^{n} a_{i j} x_{j}(t)\right. \\
& \left.+\sum_{j=1}^{n} b_{i j} x_{j}(t-\tau)\right), \quad i=1, \ldots, n,
\end{aligned}
$$

be given. The system is a generalized Lotka-Volterra model describing interaction of species in a biological community, see [Hofbauer and Sigmund, 1998; Kaszkurewicz and Bhaya, 1999; Fan and Wang, 2000; Aleksandrov, Aleksandrova and Platonov, 2013]. Here $x_{i}(t)$ is the population density of the $i$ th species, $c_{i}$, $a_{i j}, b_{i j}$ are constant coefficients, $\tau$ is a constant nonnegative delay. The coefficients $c_{i}$ characterize the intrinsic growth rate of the $i$ th population, the selfinteraction terms $a_{i i} x_{i}^{2}(t)$ and $b_{i i} x_{i}(t) x_{i}(t-\tau)$ with $a_{i i} \leq 0, b_{i i} \leq 0$ reflect the limited resources available in the environment, the terms $a_{i j} x_{i}(t) x_{j}(t)$ and $b_{i j} x_{i}(t) x_{j}(t-\tau)$ for $j \neq i$ describe the influence of population $j$ on population $i$.

Let $\mathbb{R}_{+}^{n}$ be the nonnegative cone of the space $\mathbb{R}^{n}$ : $\mathbb{R}_{+}^{n}:=\left\{x \in \mathbb{R}^{n} \mid x \geq 0\right\}$, and int $\mathbb{R}_{+}^{n}$ be the interior of $\mathbb{R}_{+}^{n}$. It should be noted that int $\mathbb{R}_{+}^{n}$ is an invariant set for (8). For biological reasons, we will consider this system with respect to the state space int $\mathbb{R}_{+}^{n}$.

Let $A$ and $B$ denote the matrices $A=\left\{a_{i j}\right\}_{i, j=1}^{n}$, $B=\left\{b_{i j}\right\}_{i, j=1}^{n}$. We consider the case where $n$ is an even number ( $n=2 k, k$ is a positive integer) and the matrices $A$ and $B$ have the form (3) and (4), respectively. Thus, interactions between populations with numbers $2 s-1$ and $2 s$ are competition, predation or symbiosis type, whereas interactions between populations with numbers $2 s$ and $2 s+1$ are commensalism or amensalism type (see [Begon, Harper and Townsend, 1996; Hofbauer and Sigmund, 1998]), $s=1, \ldots, k$, and $x_{n+1}(t)=x_{1}(t)$. Moreover, we assume that there is a delay in interactions of commensalism and amensalism type.

Theorem 2. If the system (8) admits an equilibrium position $\tilde{x} \in \operatorname{int} \mathbb{R}_{+}^{n}$, then, under the conditions (6) and (7), the equilibrium position is globally asymptotically stable in int $\mathbb{R}_{+}^{n}$ for any value of the delay $\tau$.

Proof. If $\tilde{x}=\left(\tilde{x}_{1}, \ldots, \tilde{x}_{n}\right)^{T} \in \operatorname{int} \mathbb{R}_{+}^{n}$ is an equilibrium position of (8), then the system can be rewritten as follows

$$
\begin{aligned}
& \dot{x}_{i}(t)=x_{i}(t)\left(\sum_{j=1}^{n} a_{i j}\left(x_{j}(t)-\tilde{x}_{j}\right)\right. \\
& \left.+\sum_{j=1}^{n} b_{i j}\left(x_{j}(t-\tau)-\tilde{x}_{j}\right)\right), \quad i=1, \ldots, n .
\end{aligned}
$$

Assume that the inequalities (6) and (7) are fulfilled. Hence (see Theorem 1), there exist diagonal positive definite matrices $P$ and $Q$ such the matrix (2) is negative definite.

Choose a Lyapunov-Krasovskii functional for (9) in the form

$$
\begin{aligned}
& V\left(x_{t}\right)=2 \sum_{i=1}^{n} p_{i}\left(x_{i}(t)-\tilde{x}_{i}-\tilde{x}_{i} \log \frac{x_{i}(t)}{\tilde{x}_{i}}\right) \\
& +\sum_{i=1}^{n} q_{i} \int_{-\tau}^{0}\left(x_{i}(\theta)-\tilde{x}_{i}\right)^{2} d \theta
\end{aligned}
$$

where positive coefficients $p_{i}$ and $q_{i}$ are diagonal entries of the matrices $P$ and $Q$.

Taking into account positive definiteness of the matrix (2) and using the Schur complement, see Theorem 7.7.6 in [Horn and Johnson, 1985], we obtain that the derivative of $V\left(x_{t}\right)$ with respect to (9) satisfies the estimate

$\dot{V}\left(x_{t}\right) \leq-\alpha \sum_{i=1}^{n}\left(\left(x_{i}(t)-\tilde{x}_{i}\right)^{2}+\left(x_{i}(t-\tau)-\tilde{x}_{i}\right)^{2}\right)$

for all $x_{i}(t), x_{i}(t-\tau) \in \operatorname{int} \mathbb{R}_{+}^{n}$. Here $\alpha$ is a positive constant. This completes the proof.

\section{Delay-Independent Stability Conditions for a Mechanical System}

Next, consider the system

$$
\begin{gathered}
\ddot{x}_{1}(t)+a_{1} \dot{x}_{1}(t)+c_{1} x_{1}(t)=b_{1} x_{n}(t-\tau), \\
\ddot{x}_{j}(t)+a_{j} \dot{x}_{j}(t)+c_{j} x_{j}(t)=b_{j} x_{j-1}(t-\tau), \\
j=2, \ldots, k .
\end{gathered}
$$

Here $x_{s}(t) \in \mathbb{R}, a_{s}, b_{s}, c_{s}$ are constant coefficients, $s=1, \ldots, k$, and $\tau$ is a constant nonnegative delay.

Thus, (10) can be treated as a complex system describing interaction of $k$ mechanical systems with one degree of freedom.

Assume that $a_{s}>0, c_{s}>0, s=1, \ldots, k$. Under this assumption, the isolated delay-free systems

$$
\ddot{x}_{s}(t)+a_{s} \dot{x}_{s}(t)+c_{s} x_{s}(t)=0, \quad s=1, \ldots, k,
$$


are asymptotically stable, and the coefficients $a_{s}$ and $c_{s}$ can be considered as damping and stiffness ratios, respectively.

We will look for delay-independent asymptotic stability conditions for the complex system (10).

With the aid of the substitution $z_{2 s-1}(t)=\lambda_{s} x_{s}(t)+$ $\dot{x}_{s}(t), z_{2 s}(t)=x_{s}(t), s=1, \ldots, k$, transform (10) to the system

$$
\dot{z}(t)=A z(t)+B z(t-\tau) .
$$

Here $z(t)=\left(z_{1}(t), \ldots, z_{n}(t)\right)^{T}, n=2 k, \lambda_{s}$ are auxiliary parameters, matrices $A$ and $B$ have the form (3) and (4), respectively, with

$$
\begin{aligned}
& a_{2 s-12 s-1}=\lambda_{s}-a_{s}, a_{2 s-12 s}=\lambda_{s}\left(a_{s}-\lambda_{s}\right)-c_{s}, \\
& a_{2 s 2 s-1}=1, \quad a_{2 s 2 s}=-\lambda_{s}, \quad s=1, \ldots, k .
\end{aligned}
$$

Let

$$
0<\lambda_{s}<a_{s}, \quad s=1, \ldots, k .
$$

Then inequalities (6) are fulfilled for entries of the matrix $A$.

Applying Theorem 1, we obtain that, under the conditions (12) and

$$
\mu_{1} \ldots \mu_{k}>\left|b_{1} \ldots b_{k}\right|
$$

the system (11) is diagonally Riccati stable. Here

$$
\mu_{s}=\left\{\begin{array}{l}
c_{s} \text { for } \beta_{s}>c_{s} / 2 \\
2 \sqrt{\beta_{s}\left(c_{s}-\beta_{s}\right)} \text { for } \beta_{s} \leq c_{s} / 2
\end{array}\right.
$$

and $\beta_{s}=\lambda_{s}\left(a_{s}-\lambda_{s}\right), s=1, \ldots, k$.

From (12) it follows that $\beta_{s} \in\left(0, a_{s}^{2} / 4\right), s=$ $1, \ldots, k$.

Find $\beta_{1}, \ldots, \beta_{k}$ for which the condition (13) defines the largest domain of values for the parameters $a_{s}, b_{s}, c_{s}$. It is easy to verify that we should take $\beta_{s}=c_{s} / 2$ for $a_{s}^{2}>2 c_{s}$, and $\beta_{s} \rightarrow a_{s}^{2} / 4-0$ for $a_{s}^{2} \leq 2 c_{s}$.

Thus, the following theorem is valid.

Theorem 3. Let $a_{s}>0, c_{s}>0, s=1, \ldots, k$. If

$$
\sigma_{1} \ldots \sigma_{k}>\left|b_{1} \ldots b_{k}\right|
$$

where

$$
\sigma_{s}=\left\{\begin{array}{l}
c_{s} \text { for } a_{s}^{2}>2 c_{s}, \\
a_{s} \sqrt{c_{s}-a_{s}^{2} / 4} \text { for } a_{s}^{2} \leq 2 c_{s},
\end{array} \quad s=1, \ldots, k\right.
$$

then the system (10) is asymptotically stable for any value of the delay $\tau$.

Remark 3. The condition (14) determines, how small the coefficients characterizing the connections between the subsystems should be compared with the parameters of the isolated subsystems, in order to guarantee the delay-independent asymptotic stability of (10).

Remark 4. If, for some $r \in\{1, \ldots, k\}$, the corresponding damping coefficient $a_{r}$ is sufficiently large $\left(a_{r}^{2} \geq 2 c_{r}\right)$, then the condition (14) is independent of the coefficient.

In particular, we obtain the following corollary.

Corollary 1. Let $a_{s}^{2} \geq 2 c_{s}, s=1, \ldots, k$. Then, under the condition $c_{1} \ldots c_{k}>\left|b_{1} \ldots b_{k}\right|$, the system (10) is asymptotically stable for any value of the delay $\tau$.

\section{A Problem of Formation Control}

In recent years, the problem of formation control of multiagent systems has attracted considerable attention due to its broad applications, see [Bullo, Cortes and Martinez, 2009; Ren and Cao, 2011; Parsegov, Polyakov and Shcherbakov, 2012; Proskurnikov and Parsegov, 2016; Frolov, Koronovskii, Makarov, Maksimenko, Goremyko and Hramov, 2017].

One of the simplest formation control problems is related to equidistant deployment of agents on a line segment. Some approaches to the solution of the problem were proposed in [Wagner and Bruckstein, 1997; Kvinto and Parsegov, 2012; Parsegov, Polyakov and Shcherbakov, 2012; Proskurnikov and Parsegov, 2016]. In the papers [Wagner and Bruckstein, 1997; Parsegov, Polyakov and Shcherbakov, 2012] the protocols were designed providing equidistant distribution for agents modeled as the first order integrators. In particular, in [Parsegov, Polyakov and Shcherbakov, 2012], conditions of fixed-time stability of such systems were derived. In [Kvinto and Parsegov, 2012; Proskurnikov and Parsegov, 2016], the case was considered where agent dynamics are described by the double integrators. However, it should be noted that in [Wagner and Bruckstein, 1997; Kvinto and Parsegov, 2012; Parsegov, Polyakov and Shcherbakov, 2012; Proskurnikov and Parsegov, 2016] it was assumed that there are no communication delays in the investigated multiagent systems.

Let us show that the results of the present paper can be used in the problem of equidistant deployment of agents on a line segment under protocols with communication delays.

Consider a group of $k$ mobile agents on the line. Let $x_{s}(t) \in \mathbb{R}$ be the position of the $s$ th agent at time $t \geq 0$, $s=1, \ldots, k$. Assume that the dynamics of agents are described by the double integrators

$$
\ddot{x}_{s}(t)+a \dot{x}_{s}(t)=u_{s}, \quad s=1, \ldots, k .
$$


Here $u_{s} \in \mathbb{R}$ is the control input, $a$ is a constant positive damping coefficient.

Let a segment $\left[x_{b}, x_{e}\right]$ be given. The problem is to design a feedback control protocol providing the equidistant distribution of the agents on the segment for $t \rightarrow$ $\infty$ and any initial conditions.

Such a problem was studied in [Kvinto and Parsegov, 2012; Proskurnikov and Parsegov, 2016]. It was assumed that each agent receives information about the distances between itself and its nearest left and right neighbors, i.e., the agent $x_{s}$ receives information about the distances $x_{s-1}(t)-x_{s}(t)$ and $x_{s+1}(t)-x_{s}(t)$, $s=1, \ldots, k$, where $x_{0}(t)=x_{b}, x_{k+1}(t)=x_{e}$.

In this section, we will study the problem of equidistant deployment of agents under the following assumptions.

Assumption 1. The agent $x_{1}$ knows the total number of agents in the system, but it doesn't know the length of the interval $\left[x_{b}, x_{e}\right]$. In addition, it receives information about the distances $x_{1}(t)-x_{b}$ and $x_{1}(t)-x_{k}(t-$ $\tau)$, where $\tau$ is a constant nonnegative delay.

Assumption 2. For each $j \in\{2, \ldots, k\}$, the agent $x_{j}$ knows the desired final distance $\Delta$ between agents $\left(\Delta=\left(x_{e}-x_{b}\right) /(k+1)\right)$, but it knows neither the total number of agents in the system nor the length of the interval $\left[x_{b}, x_{e}\right]$. In addition, it receives information about the distance $x_{j}(t)-x_{j-1}(t-\tau)$.

Under Assumptions 1 and 2, one can use the protocol

$$
\begin{aligned}
& u_{1}=\frac{x_{k}(t-\tau)-x_{1}(t)}{k-1}+x_{b}-x_{1}(t), \\
& u_{j}=x_{j-1}(t-\tau)-x_{j}(t)+\Delta, j=2, \ldots, k .
\end{aligned}
$$

Substituting (16) into (15), we obtain the closed-loop system

$$
\begin{aligned}
& \ddot{x}_{1}(t)+a \dot{x}_{1}(t)=\frac{x_{k}(t-\tau)-x_{1}(t)}{k-1} \\
& \quad+x_{b}-x_{1}(t), \\
& \ddot{x}_{j}(t)+a \dot{x}_{j}(t)=x_{j-1}(t-\tau)-x_{j}(t)+\Delta, \\
& \quad j=2, \ldots, k .
\end{aligned}
$$

It is easy to verify that (17) admits the equilibrium position $\tilde{x}=\left(\tilde{x}_{1}, \ldots, \tilde{x}_{k}\right)^{T}$, where $\tilde{x}_{s}=x_{b}+s \Delta, s=$ $1, \ldots, k$. Hence, the equilibrium position corresponds to the equidistant distribution of agents on the segment $\left[x_{b}, x_{e}\right]$.

With the aid of the transformation $y(t)=x(t)-\tilde{x}$, we arrive at the system

$$
\begin{gathered}
\ddot{y}_{1}(t)+a \dot{y}_{1}(t)=\frac{y_{k}(t-\tau)-y_{1}(t)}{k-1}-y_{1}(t), \\
\ddot{y}_{j}(t)+a \dot{y}_{j}(t)=y_{j-1}(t-\tau)-y_{j}(t), \\
j=2, \ldots, k .
\end{gathered}
$$

It should be noted that (18) is a special case of the system (10). Here $a_{s}=a, s=1, \ldots, k, c_{1}=k /(k-$ $1), b_{1}=1 /(k-1), c_{j}=b_{j}=1, j=2, \ldots, k$.

Applying Theorem 3, we obtain that the following theorem is valid.

Theorem 4. If one of the conditions

(i) $a \geq \sqrt{2}$;

(ii) $0<a<\sqrt{2}$ and

$$
a^{2 k}(k-1)^{2}\left(1-\frac{a^{2}}{4}\right)^{k-1}\left(\frac{k}{k-1}-\frac{a^{2}}{4}\right)>1
$$

holds, then the equilibrium position $\tilde{x}$ of (17) is asymptotically stable for any nonnegative delay $\tau$.

\section{Simulation Results}

To illustrate the effectiveness of the proposed approaches, consider a group consisting of five agents with the integrator dynamics (17). Let $\left[x_{b}, x_{e}\right]=[0,1]$.

For simulation, we assume that $a=1, \tau=2$ and $x(t)=(0.1,0.4667,0.7,0.2,0.5)^{T}$ for $t \in[-2,0]$.

Figure 2 demonstrates the convergence of agents to the equidistant distribution.

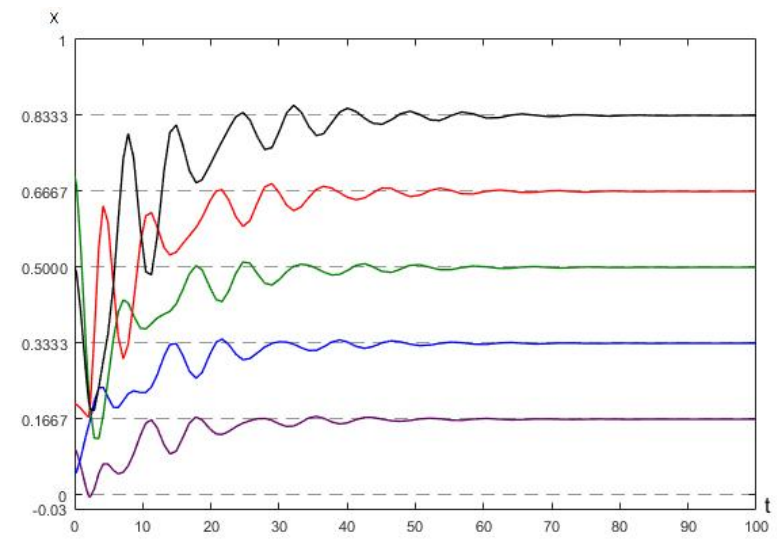

Figure 2. The agent time history $(a=1, \tau=2)$ 


\section{Conclusion}

In the present paper, necessary and sufficient conditions of the diagonal Riccati stability are derived for a complex time-delay system with a special structure of connections. The fulfilment of the conditions implies delay-independent asymptotic stability of the zero solution of the considered system. Some applications of the obtained results are presented.

Future research aims at constructing diagonal Lyapunov-Krasovskii functionals for complex systems with delay and switched connections.

\section{Acknowledgements}

The reported study was partially financially supported by the Russian Foundation for Basic Research (Grant No. 17-01-00672-a).

\section{References}

Aleksandrov, A.Yu., Aleksandrova, E.B., and Platonov, A.V. (2013). Ultimate boundedness conditions for a hybrid model of population dynamics. In Proc. 21 st Mediterranean Conference on Control and Automation. Platanias-Chania, Crite, Greece, June 25-28, pp. 622-627.

Aleksandrov, A., and Mason, O. (2014). Diagonal Lyapunov-Krasovskii functionals for discrete-time positive systems with delay. Syst. Control Lett., 63, pp. 63-67.

Aleksandrov, A., Mason, O. (2016). Diagonal Riccati stability and applications. Linear Algebra and its Applications, 492, pp. 38-51.

Aleksandrov, A., and Mason, O. (2018) Diagonal stability of a class of discrete-time positive switched systems with delay. IET Control Theory \& Applications, 12(6), pp. 812-818.

Aleksandrov, A., Mason, O., and Vorob'eva, A. (2017). Diagonal Riccati stability and the Hadamard product. Linear Algebra and its Applications, 534, pp. 158173.

Alyshev, A., Dudarenko, N., and Melnikov, V. (2018). Parametric identification of reaction wheel pendulums with adaptive control. Cybernetics and Physics, 7(2), pp. 57-65.

Arcat, M., and Sontag, E. (2006). Diagonal stability of a class of cyclic systems and its connection with the secant criterion. Automatica, 42(9), pp. 1531-1537.

Begon, M., Harper, J.L., and Townsend, C.R. (1996). Ecology: Individuals, Populations, and Communities. Blackwell Science Ltd. Cambridge, Massachusetts, USA.

Bullo, F., Cortes, J., and Martinez, S. (2009). Distributed Control of Robotics Networks. Princeton Univ. Press. Princeton.
Fan, M., and Wang, K. (2000). Global periodic solutions of a generalized $n$-species Gilpin-Ayala competition model. Computers and Mathematics with Applications, 40, pp. 1141-1151.

Frolov, N., Koronovskii, A, Makarov, V., Maksimenko, V., Goremyko, M., and Hramov, A. (2017). Control of pattern formation in complex networks by multiplexity. Cybernetics and Physics, 6(3), pp. 121125.

Hofbauer, J., and Sigmund, K. (1998). Evolutionary Games and Population Dynamics. Cambridge University Press, Cambridge.

Horn, R.A., and Johnson, C.R. (1985). Matrix Analysis. Cambridge University Press, New York.

Kaszkurewicz, E., and Bhaya, A. (1999). Matrix Diagonal Stability in Systems and Computation. Birkhuser, Boston, Basel, Berlin.

Kraaijevanger, J.F.B. (1991). A characterisation of Lyapunov diagonal stability using Hadamard products. Linear Algebra and its Applications, 151, pp. 245254.

Kvinto, Ya.I., and Parsegov, S.E. (2012). Equidistant arrangement of agents on line: Analysis of the algorithm and its generalization. Automation and Remote Control, 73(11), pp. 1784-1793.

Mason, O. (2012). Diagonal Riccati stability and positive time-delay systems. Syst. Control Lett., 61, pp. 610.

Mason, O. and Shorten, R. (2006). On the simultaneous diagonal stability of a pair of positive linear systems. Linear Algebra and its Applications, 413, pp. 13-23.

Parsegov, S.E., Polyakov, A.E., and Shcherbakov, P.S. (2012). Nonlinear fixed-time control protocol for uniform allocation of agents on a segment. In Proc. 51st IEEE Conf. Decision and Control. Maui, Hawaii, USA, Dec. 10-13, pp. 7732-7737.

Proskurnikov, A.V., and Parsegov, S.E. (2016). Problem of uniform deployment on a line segment for second-order agents. Automation and Remote Control, 77(7), pp. 1248-1258.

Ren, W. and Cao, W. (2011). Distributed Coordination of Multi-Agent Networks. Springer-Verlag, London.

Shorten, R. and Narendra, K. (2009). On a theorem of Redheffer concerning diagonal stability. Linear Algebra and its Applications, 431, pp. 2317-2329.

Talagaev, Y. (2017). State estimation, robust properties and stabilization of positive linear systems with superstability constraints. Cybernetics and Physics, 6(1), pp. 32-39.

Wagner, I. and Bruckstein, A.M. (1997). Row straightening by local interactions. Circuits, Systems and Signal Processing, 16(3), pp. 287-305. 